\title{
Infectious arteritis of the femoral artery as a cause of recurrent hemorrhage after endovascular treatment of thrombosed popliteal artery aneurysm
}

\author{
Witold Woźniak, Piotr Ciostek \\ First Department of General and Vascular Surgery, Faculty of Medicine, Medical University of Warsaw, Poland
}

\begin{abstract}
Due to a great number of endovascular interventions, conducted over the last decades, a huge percentage of obese patients and administration of double antiplatelet therapy, manual compression (MC) is more and more frequently replaced by a vascular closure device of which the most common is Angio-Seal. The study presents a case of a 63-year-old female patient with recurrent hemorrhages of the common femoral artery (CFA), originally closed after an endovascular intervention of a popliteal artery aneurysm with the use of Angio-Seal VIP. After around 3 weeks following the original surgery, the patient at first developed a pseudoaneurysm and then septic inflammation of the femoral artery, manifested with recurrent hemorrhages from the groin. The patient was operated on many times and an implantation of an ilio-femoral bypass from the femoral vein turned out to be an effective solution. Staphylococcus epidermidis MRSA was cultured in the femoral artery wall and a histopathological examination confirmed infectious arteritis. The presented case of a rare septic complication after the application of a closure device shows that it is essential to carefully monitor surgical approach areas in patients who are quickly discharged from hospital after surgical interventions, to select treatment methods tailored for individual patients as well as implement particular surgical management.
\end{abstract}

Key words: Angio-Seal, infectious complications, pseudoaneurysm, vascular closure device, popliteal artery aneurysm

Acta Angiol 2020; 26, 3: II3-117

\section{Introduction}

Manual compression (MC) is a basic hemostatic method after an arterial puncture in endovascular surgery. However, a rapid increase in the number of endovascular interventions over the last few decades, a huge percentage of obese patients, administration of double antiplatelet therapy as well as the fact $M C$ is a disadvantage for both medical personnel and patients, led to a more frequent replacement of $M C$ with a vascular closure device (VCDs). VCDs, introduced at the beginning of the 1990s, considerably shorten the time needed to stop bleeding after performing an arterial puncture [I]. The percentage of complications after application of VCDs in comparison to MC is different. Some previous studies revealed that these devices significantly decrease the number of complications [2]. However, authors of recently published analyses claim that the number of VCDs- and MC-related complications is similar [I]. Besides, Angio-Seal VIP device was introduced into practice more than 20 years ago [3]. It allows closing arteriotomy between a polymer anchor and a plug of collagen sponge which are self-tightening. The anchor is placed in the arterial lumen and the collagen plug - outside. Hemostasis is achieved by a mechanical pressure and stimulation of coagulation 
with collagen. All elements of the Angio-Seal VIP set are absorbable [4]. Potential complications related to application of Angio-Seal VIP can be divided into three main groups: ischemic (stenosis/occlusion of the access artery, distal embolization), hemorrhagic (bleeding, hematoma, pseudoaneurysm) and infectious (foreign body reaction, infection) [5].

The article presents a case of a female patient with recurrent hemorrhages of the common femoral artery (CFA), originally closed after an endovascular intervention with the use of Angio-Seal VIP device.

\section{Case report}

A 63-year-old patient, hypertensive, with insulindependent diabetes, chronic renal failure (on admission serum creatinine was $1.69 \mathrm{mg} \%$ ), after cerebral stroke, was admitted to the clinic due to critical ischemia of the left lower limb. Imaging examinations (DUS - duplex doppler ultrasound, CTA - computed tomography angiography) revealed on the left side a thrombosed aneurysm of the popliteal artery (PA), up to $24 \mathrm{~mm}$ in diameter and occluded anterior and posterior tibial arteries. The patient was qualified for endovascular treatment. Until the surgery, the patient had been undergoing antithrombotic treatment, involving administration of low molecular weight heparin (Clexane $80 \mathrm{mg}$ every $12 \mathrm{~h}$ ). Through a puncture in the common femoral artery (CFA) on the right side, conducted with the cross-over technique, 5F PIG Impress catheter was inserted to the external iliac artery (EIA) on Lake Region Medical Starter Guidewire 0.035. Besides, arteriography was performed. It confirmed a thrombosed aneurysm of the left PA, with a narrow fibular artery (anterior and posterior tibial arteries - occluded). After getting through the occluded PA with the use of the mentioned catheter and $5 F$ VERT Impress catheter, 4F Cragg-Mcnamara Infusion Catheter $100 \times 20$ was inserted. Its end was placed in the fibular artery. Intra-arterial administration of $(50 \mathrm{mg} / 50 \mathrm{~mL})$ with an infusion pump $(1 \mathrm{~mL} / \mathrm{h})$ was continued for $48 \mathrm{~h}$, regularly every $6 \mathrm{~h}$, monitoring fibrinogen. Simultaneously, the vascular sheath was rinsed with solution of unfractioned heparin $(25 \mathrm{mg} / 50 \mathrm{~mL})$, also administered from an infusion pump $(500 \mathrm{U} / \mathrm{h})$. After $24 \mathrm{~h}$, the thrombolytic process was controlled in angiography which confirmed that a huge amount of embolic material is still observed. Only next angiography, conducted after 48 hours, revealed a patent canal of PA aneurysm and a patent tibial artery. A covered stent Gore Viabahn Endoprosthesis $8 \times 150 \mathrm{~mm}$ was implanted into PA, modeling it with Dorado balloon $7 \times 150 \mathrm{~mm}$, Bard. After achieving a good morphological and hemodynamic effect, the approach area in the right CFA (after sheath $8 \mathrm{~F}$ ) was secured with Angio-Seal

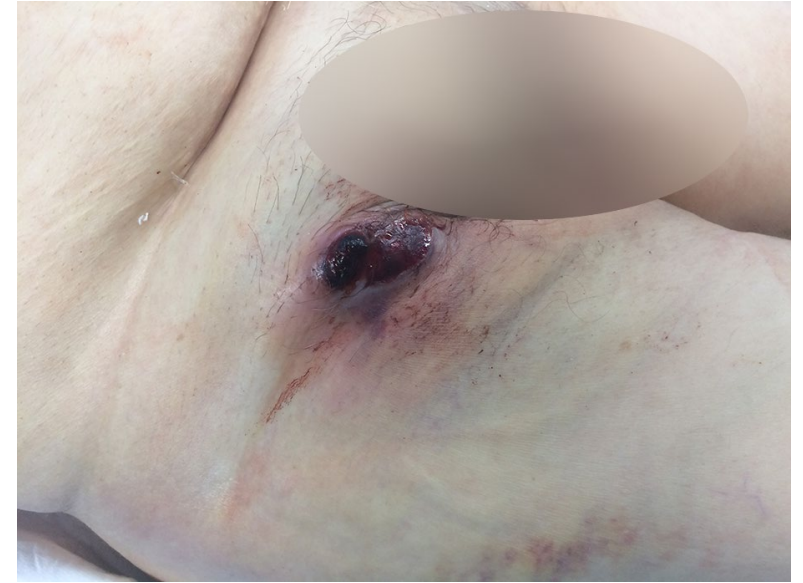

Figure I. Ruptured common femoral artery pseudoaneurysm in the right groin

VIP device. An antibiotic (Biofazolin I g every $12 \mathrm{~h}$ ) was administered during the thrombolytic treatment. The course of the surgery was uncomplicated; on day 3 following the surgery, the patient' pulse on both PAs was regular and she did not demonstrate any hematoma or aneurysm in the right groin so she was discharged home and ordered a double antiplatelet therapy (ASA $75 \mathrm{mg}$ daily and clopidogrel $75 \mathrm{mg}$ daily). Five days following the discharge, the patient was routinely examined in the outpatient clinic. The pulse on both PAs was regular and no hematoma or clinical manifestations of pseudoaneurysm were observed.

On day 19 following the surgery, the patient was readmitted to the clinic with symptoms of ruptured aneurysm in the right groin, accompanied by skin necrosis above the aneurysm and elevated inflammatory parameters (leukocytosis $25.500 / \mathrm{mm}^{3}, \mathrm{RCPR} 173.7 \mathrm{mg} \%$ ) (Fig. I). The patient underwent an emergency operation. The visualized anterior wall of CFA appeared to be damaged. There was a hole $3 \mathrm{~mm}$ in diameter, which was closed with two single sutures (Prolene 5/0). Next, the aneurysm was removed and some considerably inflamed subcutaneous and dermal tissue was resected (culture was also collected). After the operation, the patient was initiated empirical intravenous antibiotic therapy (Biotraxon $2 \mathrm{~g}$ every $12 \mathrm{~h}$ and Metronidazole $0.5 \mathrm{~g}$ every $8 \mathrm{~h}$ ). After obtaining culture results (Proteus vulgaris, Staphylococcus aureus MSSA), this therapy was replaced with a targeted antibiotic therapy (Ciprinol $0.2 \mathrm{~g}$ every $12 \mathrm{~h}$ ).

On day 27 , an inguinal hemorrhage occurred. During a reoperation, the doctors observed suture dehiscence and a hole on the anterior wall of CFA, $8 \mathrm{~mm}$ in diameter. The damaged fragment of the CFA anterior wall was resected and the defect was closed with a saphenous 
vein patch. The sample of the damaged wall was sent to a bacteriological analysis (negative culture).

On day 33 , the patient suffered another hemorrhage from the groin. During a reoperation, the doctors observed suture dehiscence between the medial edge of the patch and the CFA wall. The damaged fragment of the CFA was resected and a $4-\mathrm{cm}$ saphenous vein graft, collected from the left groin, was implanted. The lower anastomosis of the graft was performed about $3 \mathrm{~mm}$ from the ostium of the deep femoral artery (PFA). The removed fragment of CFA was sent to a bacteriological analysis but the culture appeared to be negative. A control CTA revealed huge inflamed infiltration, modeling the course of CFA and the implanted graft and no manifestations of aneurysm. The patient with a post-operative wound, partially healing by granulation, was discharged home (culture from the wound collected a week prior to the discharge, turned out to be negative).

On day 75 , the patient was readmitted due to another inguinal hemorrhage. CTA visualized aneurysm $76 \mathrm{~mm}$ in diameter, adjacent to the right CFA (Fig. 2). Another operation revealed that CFA wall, the implanted graft and the PFA ostium are completely damaged. Since PFA walls were inflamed and fragile, the artery was punctured. Next, a I5-cm ilio-femoral bypass, made of the femoral vein (collected from the right lower limb), was implanted. An upper anastomosis was performed around $5 \mathrm{~cm}$ above the inguinal fold, whereas a lower anastomosis was performed in the upper $\mathrm{I} / 3$ of the thigh (the surgeons attempted to make both the anastomoses within uninflamed tissue). The resected fragment of CFA was again sent to a microbiological and histopathological analysis. This time, the culture appeared to contain Staphylococcus epidermidis MRSA (MLSB), which, according to an antibiogram, was managed with Vancomycin $0.5 \mathrm{~g}$, administered every 6h. A histopathological examination confirmed arteritis (Fig. 3). The post-operative course was uncomplicated. The patient's pulse on both PAs was regular and the wound healed so she was discharged home. Follow-up examinations on days 7 and 30 showed that the wound was healed and the pulse was present on both PAs.

On day 166, the patient was readmitted to the clinic due to rest pains of the right limb which had lasted for almost $24 \mathrm{~h}$. CTA (performed two weeks before) confirmed a critical stenosis of the right EIA (at the level of the upper anastomosis). The patient was qualified for endovascular treatment. By puncturing the left CFA with the cross-over technique, the surgeon inserted $5 \mathrm{~F}$ PIG Impress catheter to the right common iliac artery (CIA) on Lake Region Medical Starter Guidewire 0.035". Arteriography visualized occluded EIA, the ilio-femoral bypass and the initial segment of the superficial femoral

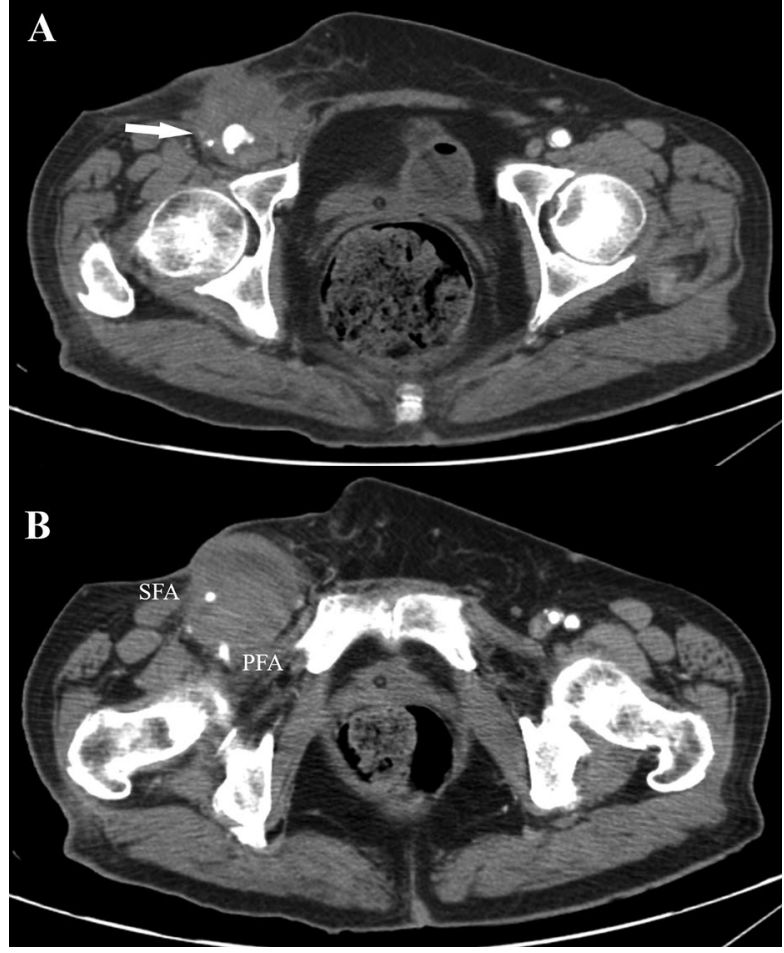

Figure 2. CT shows large common artery pseudoaneurysm. A - CT scan at the level of CFA (arrow shows CFA with visible leakage of contrast); $\mathbf{B}-C T$ scan at level of $\mathrm{I}-2 \mathrm{~cm}$ below the division of CFA into SFA and PFA (massive aneurysm shifts both femoral arteries to the side)

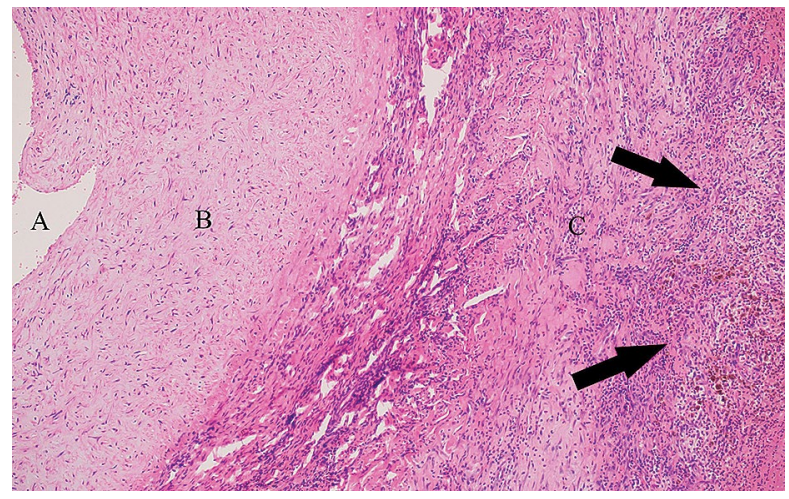

Figure 3. Histopathological examination of the common femoral artery wall: A - artery lumen; B - Tunica media; C - Tunica adventitia. Arrows show inflammatory infiltrate from lymphocytes and granulocytes.

artery (SFA) (Fig. 4A). With the use of the mentioned guidewire and 5F VERT Impress $125 \mathrm{~cm} 0.035$ " catheter, the author managed to get through the occlusion and placed the end of the guidewire in PA. Next percutaneous transluminal angioplasty (PTA) with the application of Dorado balloon $5 \times 150 \mathrm{~mm}$, Bard of 
the bypass and SFA was performed. The balloon angioplasty was accompanied by implantation of two stents Everflex, going from top $7 \times 120 \mathrm{~mm}$ and $6 \times 150 \mathrm{~mm}$. A follow-up angiography showed stenosis above the upper stent. Hence, the stent was extended upwards with the third stent - Boston Express LD Vascular $7 \times 27 \mathrm{~mm}$. After achieving a good hemodynamic effect, the approach in the right CFA was closed with MC (Fig. 4B). The post-operative course was uncomplicated; on day 3 following the intervention, the patient did not demonstrate clinical signs of hematoma or aneurysm in the left groin and her pulse on both PAs was regular. She was discharged home with recommendation of double antiplatelet therapy. Follow-up examinations were conducted in an outpatient clinic on days 7 and 30 and next, every month for half a year. During this time, no lower limb ischemia or suspicion of aneurysm were observed. CTA (2 months after the last operation) did not show significant stenosis or any other abnormalities, requiring an intervention (Fig. 5).

\section{Discussion}

Angio-Seal studies report a rate of hemorrhagic complications of $3.8-7.7 \%$ and hematomas resulting from these complications may potentially contribute to infectious complications [6, 7]. The risk of infection of the approach definitely increases in the event of a foreign body. Elements of VCD are such a foreign body. Despite this, the risk of infectious complications after application of Angio-Seal is still low. If such complications occur, they are usually mild and rare (0.3\%) [8]. An infection of the arterial wall in the approach area poses a far more serious threat. It subsequently leads to a septic form of arteritis which is particularly resistant to pharmacotherapy. Frazee and Flaherty [9] presented 10 cases of septic arteritis which occurred after repeat PTCA and were related to recatheterization of the original approach area. In all patients, Staphylococcus aureus was the etiological factor. In six cases, aneurysm occurred. It was surgically treated by resection of a fragment of FA with implantation of a saphenous vein bypass. In our patient, culture of aneurysm appeared to contain Staphylococcus aureus MSSA, whereas in the culture of the FA wall contained Staphylococcus epidermidis MRSE/MLSB. What is interesting, a positive result of the culture of the arterial wall was obtained only in the third test, during the fourth surgical intervention. Despite initiating targeted therapy after the first two operations, no clinical improvement was observed. Minor surgical techniques (a vascular patch, a short saphenous vein graft) appeared to be ineffective, either. Only a reconstructive surgery, consisting in a complete resection of CFA and replacing it with a femoral vein bypass, implanted from

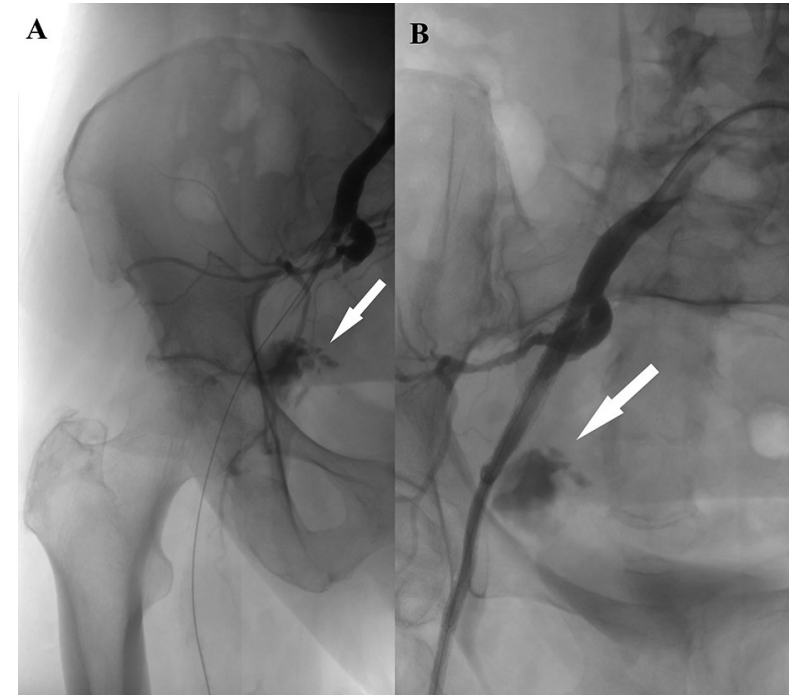

Figure 4. Arteriography 3 month after implantation of ilio-femoral bypass. A - occlusion of external iliac artery, ilio-femoral bypass and common femoral artery; B - final arteriography after angioplasty and stenting (arrows show extravasation after lesion collateral artery)

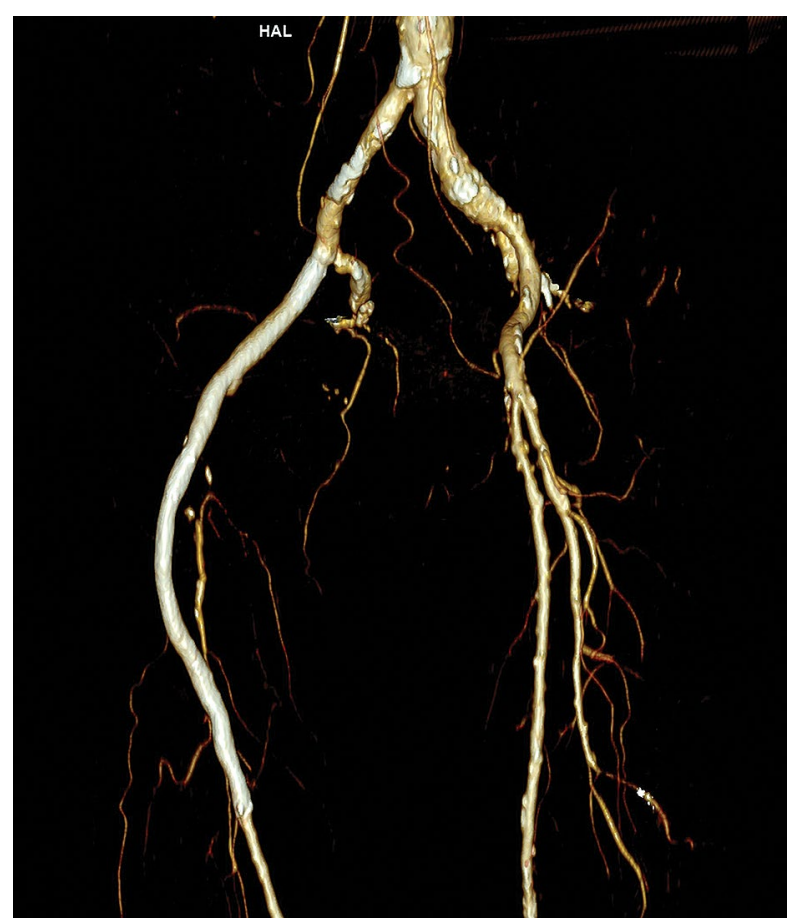

Figure 5. CTA 2 month after angioplasty shows good hemodynamic effect

EIA to SFA, where both anastomoses were performed in health tissue, brought expected clinical effect. Severe infections after administration of Angio-Seal occur rarely. The Medline database reports single cases [10]. 
Copper and Miller [ $\mathrm{I}$ I] presented one case of confirmed endartertis in a patient after application of Angio-Seal, where Staphylococcus aureus was the etiological factor. The patient was operated on, had CFA and its bifurcation resected. He was also implanted a femoro-femoral suprapubic bypass (from the left CFA to the right SFA) from a Dacron prosthesis. Such solution seems to be risky because implantation of an artificial prosthesis in infected environment might contribute to development of another infection. However, distant prognosis of that patient was not given. On the other hand, the adopted solution decreased the risk of restenosis in the place of anastomosis, an incident which was observed in our patient (stenosis of anastomosis of the femoral vein and the iliac artery). An important question arises: How to avoid such complications in the future? If, according to the instruction, the sheath is placed for longer than $8 \mathrm{~h}$, antibiotics should be applied, which, by the way, was done. The manufacturer of Angio-Seal recommends taking precautions and safety measures in patients who have undergone thrombolytic treatment, which was done as the patient was much more often monitored than other patients. However, she got many infections. The presented case of a rare septic complication after an application of a closure device shows that it is essential to carefully monitor surgical approaches in patients who are quickly discharged from hospital after surgical interventions, to select treatment methods tailored for individual patients as well as implement particular surgical management.

\section{Conflict of interest}

None.

\section{References:}

I. lannaccone M, Saint-Hilary G, Menardi D, et al. Network meta-analysis of studies comparing closure devices for femoral access after percutaneous coronary intervention. J Cardiovasc
Med (Hagerstown). 2018; 19(10): 586-596, doi: 10.2459/ JCM.0000000000000697, indexed in Pubmed: 30045086.

2. Arora N, Matheny ME, Sepke C, et al. A propensity analysis of the risk of vascular complications after cardiac catheterization procedures with the use of vascular closure devices. Am Heart J. 2007; 153(4): 606-6II, doi: 10.1016/j.ahj.2006.12.014, indexed in Pubmed: 17383300.

3. Nash JE, Evans DG. The Angio-Seal hemostatic puncture closure device. Concept and experimental results. Herz. 1999; 24(8): 597-606, doi: 10.1007/BF03044483, indexed in Pubmed: 10652672.

4. Noori VJ, Eldrup-Jørgensen J. A systematic review of vascular closure devices for femoral artery puncture sites. J Vasc Surg. 2018; 68(3): 887-899, doi: 10.1016/j.jvs.2018.05.019, indexed in Pubmed: 30146036.

5. Saleem T, Baril DT. Vascular Access Closure Devices. [Updated 2020 Jan 22]. In: StatPearls [Internet]. Treasure Island (FL): StatPearls Publishing; 2020.

6. Wu PJ, Dai YT, Kao HL, et al. Access site complications following transfemoral coronary procedures: comparison between traditional compression and angioseal vascular closure devices for haemostasis. BMC Cardiovasc Disord. 2015; 15: 34, doi: |0.| I86/s | 2872-0|5-0022-4, indexed in Pubmed: 259568|4.

7. Lucatelli P, Fanelli F, Cannavale A, et al. Angioseal VIP® vs. StarClose $\mathrm{SE}{ }^{\circledR}$ closure devices: a comparative analysis in non-cardiological procedures. J Cardiovasc Surg (Torino). 2017; 58(I): 80-86, doi: 10.23736/S002I-9509.16.07654-0, indexed in Pubmed: 24309473.

8. Carey D, Martin JR, Moore CA, et al. Complications of femoral artery closure devices. Catheter Cardiovasc Interv. 200 I; 52(I): 3-7; discussion 8, doi: 10.1002/1522-726x(200 I0I)52: I <3::aidccd I002 > 3.0.co;2-g, indexed in Pubmed: II I465 I2.

9. Frazee BW, Flaherty JP. Septic endarteritis of the femoral artery following angioplasty. Rev Infect Dis. I99 I; 13(4): 620-623, doi: 10.1093/clinids/ 13.4.620, indexed in Pubmed: 1925280.

10. Abando A, Hood D, Weaver F, et al. The use of the Angioseal device for femoral artery closure. J Vasc Surg. 2004; 40(2): 287-290, doi: 10.1016/j.jvs.2004.05.007, indexed in Pubmed: 15297822.

II. Cooper CL, Miller A. Infectious complications related to the use of the angio-seal hemostatic puncture closure device. Catheter Cardiovasc Interv. 1999; 48(3): 30I-303, doi: 10.1002/ (sici) I522-726x(1999II)48:3<30 I::aid-ccd I5>3.0.co;2-f, indexed in Pubmed: 10525234. 